\title{
An involution on the $K$-theory of bimonoidal categories with anti-involution
}

\author{
BIRGIT RICHTER
}

\begin{abstract}
We construct a combinatorially defined involution on the algebraic $K$-theory of the ring spectrum associated to a bimonoidal category with anti-involution. Particular examples of such are braided bimonoidal categories. We investigate examples such as $K(k u), K(k o)$ and Waldhausen's $A$-theory of spaces of the form $B B G$, for abelian groups $G$. We show that the involution agrees with the classical one for a bimonoidal category associated to a ring and prove that it is not trivial in the above mentioned examples.
\end{abstract}

55S25, 19D23; 19D10

\section{Introduction}

Several multiplicative cohomology theories possess a spectrum model that is the ring spectrum associated to a bimonoidal category. The passage from bimonoidal categories to spectra uses the additive structure of the bimonoidal category; its multiplication is then used to obtain the ring structure. For instance, in the case of singular cohomology with coefficients in a ring $R, H^{*}(-; R)$, we can view the ring $R$ as a discrete bimonoidal category. The associated spectrum is the Eilenberg-Mac Lane spectrum of the ring $R, H R$. In general, we denote the spectrum associated to a bimonoidal category $\mathcal{R}$ by $H \mathcal{R}$.

The main result of Baas, Dundas, Richter and Rognes [3] identifies the algebraic $K$-theory of $H \mathcal{R}$ with an algebraic $K$-theory construction defined by Baas, Dundas and Rognes [4], $\mathcal{K}(\mathcal{R})$, which uses the ring-like features of $\mathcal{R}$, namely uses addition and multiplication in $\mathcal{R}$ to build $K$-theory. We will recall the construction of $\mathcal{K}(\mathcal{R})$ in Section 2.

In some examples, one can therefore read off some extra structure on $\mathcal{K}(\mathcal{R})$ using this equivalence. For instance, if $R$ is a ring with anti-involution, then there is an involution on the $K$-theory of the ring $R$ and this yields an involution on

$$
\mathcal{K}\left(\mathcal{R}_{R}\right) \simeq K(H R) \simeq K^{f}(R)
$$


where $\mathcal{R}_{R}$ denotes the discrete category associated to the ring $R$ and $K^{f}(R)$ is the free $K$-theory of $R$. For the bipermutative category of finite dimensional complex vector spaces, $\mathcal{V}_{\mathbb{C}}$, we obtain that

$$
\mathcal{K}\left(\mathcal{V}_{\mathbb{C}}\right) \simeq K\left(H \mathcal{V}_{\mathbb{C}}\right)=K(k u)
$$

where $k u$ denotes the connective spectrum associated to complex topological $K$-theory. As complex conjugation gives rise to an action of the group of order two on $k u$, we obtain an induced action of $\mathbb{Z} / 2 \mathbb{Z}$ on $K(k u)$ and hence on $\mathcal{K}\left(\mathcal{V}_{\mathbb{C}}\right)$.

The aim of this paper is to place these two examples in a broader context and to investigate further examples. On the one hand we will construct an involution on $\mathcal{K}(\mathcal{R})$ for every strict bimonoidal category with anti-involution. Particular examples of such categories are braided bimonoidal categories. Hence in the special case where the braiding is symmetric we obtain bipermutative categories as a class of examples. We prove that in the classical case of $K$-theory of a ring with anti-involution our involution coincides with the classical one. Furthermore, we will consider bimonoidal categories with group actions and investigate how these relate to the constructed involution. We close with the example of the involution on Waldhausen's $A$-theory of a space $X$ for spaces of the form $X=B B G$ for an abelian group $G$. We show that in several cases such as $K(k o), K(k u)$ and $A(B B G)$, our involution is nontrivial.

The advantage of our construction of an involution is that it is relatively easy to describe: it is of a purely combinatorial nature that mimics the construction of the involution on the algebraic $K$-theory of rings with anti-involution.

Acknowledgements The author would like to thank Kobe University for the hospitality during her stay in March 2008 which was partially supported by Grant-in-Aid for Scientific Research (C) 19540127 of the Japan Society for the Promotion of Science. She thanks Christian Ausoni for asking a question that led to an important correction and Hannah König for spotting some annoying typos.

\section{$2 K$-theory of bimonoidal categories}

Roughly speaking, a (strict) bimonoidal category $\mathcal{R}$ is a category with two binary operations, $\otimes$ and $\oplus$, that let $\mathcal{R}$ behave like a rig - a ring without additive inverses. More precisely, for each pair of objects $A, B$ in $\mathcal{R}$ there are objects $A \oplus B$ and $A \otimes B$ in $\mathcal{R}$ and we assume strict associativity for both operations. There are objects $0_{\mathcal{R}} \in \mathcal{R}$ and $1_{\mathcal{R}} \in \mathcal{R}$ that are strictly neutral with respect to $\oplus$ resp. $\otimes$ and there are isomorphisms $c_{\oplus}^{A, B}: A \oplus B \rightarrow B \oplus A$ with $c_{\oplus}^{B, A} \circ c_{\oplus}^{A, B}=\mathrm{id}$. Everything in sight is 
natural and satisfies coherence conditions. The addition $\oplus$ and the multiplication $\otimes$ are related via distributivity laws.

The complete list of axioms can be found in Elmendorf and Mandell [11, Definition 3.3], with the slight difference that we demand the right distributivity map

$$
d_{r}: A \otimes B \oplus A^{\prime} \otimes B \rightarrow\left(A \oplus A^{\prime}\right) \otimes B
$$

to be the identity and $d_{\ell}$ to be a natural isomorphism. Similarly to May [16, VI, Proposition 3.5] one can see that every bimonoidal category is equivalent to a strict one, so there it is no loss of generality to assume strictness.

The ring-like features of bimonoidal categories allow us to consider matrices and algebraic $K$-theory of such categories. In the following we recall some definitions and results from Baas, Dundas and Rognes [4].

Definition 2.1 [4, Definition 3.2] The category of $n \times n$-matrices over $\mathcal{R}, M_{n}(\mathcal{R})$, is defined as follows. The objects of $M_{n}(\mathcal{R})$ are matrices $A=\left(A_{i, j}\right)_{i, j=1}^{n}$ of objects of $\mathcal{R}$ and morphisms from $A=\left(A_{i, j}\right)_{i, j=1}^{n}$ to $C=\left(C_{i, j}\right)_{i, j=1}^{n}$ are matrices $\phi=\left(\phi_{i, j}\right)_{i, j=1}^{n}$ where each $\phi_{i, j}$ is a morphism in $\mathcal{R}$ from $A_{i, j}$ to $C_{i, j}$.

Lemma 2.2 [4, Proposition 3.3] For a bimonoidal category $\left(R, \oplus, 0_{\mathcal{R}}, c_{\oplus}, \otimes, 1_{\mathcal{R}}\right)$ the category $M_{n}(\mathcal{R})$ is a monoidal category with respect to the matrix multiplication bifunctor

$$
\begin{aligned}
& M_{n}(\mathcal{R}) \times M_{n}(\mathcal{R}) \stackrel{\cdot}{\longrightarrow} M_{n}(\mathcal{R}) \\
&\left(A_{i, j}\right)_{i, j=1}^{n} \cdot\left(B_{i, j}\right)_{i, j=1}^{n}=\left(C_{i, j}\right)_{i, j=1}^{n} \text { with } C_{i, j}=\bigoplus_{k=1}^{n} A_{i, k} \otimes B_{k, j} .
\end{aligned}
$$

The unit of this structure is given by the unit matrix object $E_{n}$ which has $1_{\mathcal{R}}$ as diagonal entries and $0_{\mathcal{R}}$ in the other places.

In the following we will assume that the category $\mathcal{R}$ is small. As $\mathcal{R}$ is bimonoidal, its set of path components $\pi_{0}(\mathcal{R})$ has a structure of a rig, and its group completion, $\operatorname{Gr}\left(\pi_{0}(\mathcal{R})\right)=\left(-\pi_{0} \mathcal{R}\right) \pi_{0} \mathcal{R}$, is a ring.

Definition 2.3 [4, Definition 3.4] We define the monoid of invertible $n \times n$-matrices over $\pi_{0}(\mathcal{R}), \mathrm{GL}_{n}\left(\pi_{0}(\mathcal{R})\right)$, to be the $n \times n$-matrices over $\pi_{0}(\mathcal{R})$ that are invertible as matrices over $\operatorname{Gr}\left(\pi_{0}(\mathcal{R})\right)$. 
Note that $\mathrm{GL}_{n}\left(\pi_{0}(\mathcal{R})\right)$ is the pullback in the diagram

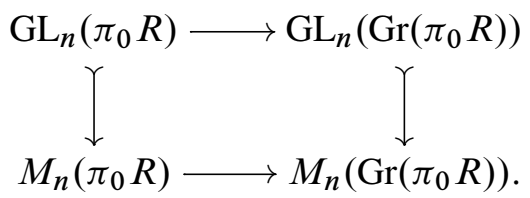

For instance, if $\pi_{0}(\mathcal{R})$ is the rig of natural numbers including zero, $\mathbb{N}_{0}$, then the elements in $\mathrm{GL}_{n}\left(\mathbb{N}_{0}\right)$ are $n \times n$-matrices over $\mathbb{N}_{0}$ that are invertible if they are considered as matrices with integral entries, ie, $\mathrm{GL}_{n}\left(\mathbb{N}_{0}\right)=M_{n}\left(\mathbb{N}_{0}\right) \cap \mathrm{GL}_{n}(\mathbb{Z})$ consists of matrices in $M_{n}\left(\mathbb{N}_{0}\right)$ with determinant \pm 1 .

Definition 2.4 [4, Definition 3.6] The category of weakly invertible $n \times n$-matrices over $\mathcal{R}, \mathrm{GL}_{n}(\mathcal{R})$, is the full subcategory of $M_{n}(\mathcal{R})$ with objects all matrices $A=$ $\left(A_{i, j}\right)_{i, j=1}^{n} \in M_{n}(\mathcal{R})$ whose matrix of $\pi_{0}$-classes $[A]=\left(\left[A_{i, j}\right]\right)_{i, j=1}^{n}$ is contained in $\mathrm{GL}_{n}\left(\pi_{0}(\mathcal{R})\right)$.

Matrix multiplication is compatible with the property of being weakly invertible and hence the category $\mathrm{GL}_{n}(\mathcal{R})$ inherits a monoidal structure from $M_{n}(\mathcal{R})$.

We recall the definition of the bar construction of monoidal categories from $[4$, Definition 3.8].

Definition 2.5 Let $(\mathcal{C}, \cdot, 1)$ be a monoidal category. The bar construction of $\mathcal{C}, B(\mathcal{C})$, is a simplicial category. Let $[q]$ be the ordered set $[q]=\{0<1<\ldots<q\}$. An object $A$ in $B_{q}(\mathcal{C})$ consists of the following data.

(a) For each $0 \leqslant i<j \leqslant q$ there is an object $A^{i j}$ in $\mathcal{C}$.

(b) For each $0 \leqslant i<j<k \leqslant q$ there is an isomorphism

$$
\phi^{i j k}: A^{i j} \cdot A^{j k} \rightarrow A^{i k}
$$

in $\mathcal{C}$ such that for all $0 \leqslant i<j<k<l \leqslant q$ the following diagram commutes:

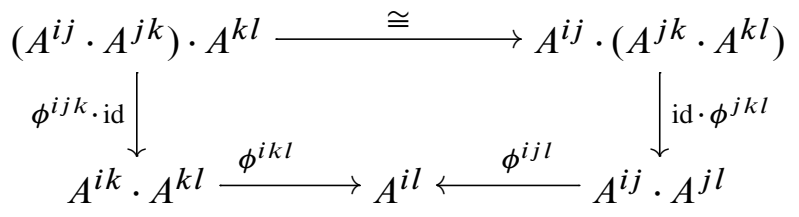

A morphism $f: A \rightarrow B$ in $B_{q} \mathcal{C}$ consists of morphisms $f^{i j}: A^{i j} \rightarrow B^{i j}$ in $\mathcal{C}$ such that for all $0 \leqslant i<j<k \leqslant q$,

$$
f^{i k} \phi^{i j k}=\psi^{i j k}\left(f^{i j} \cdot f^{j k}\right): A^{i j} \cdot A^{j k} \rightarrow B^{i k} .
$$

Here, the $\psi^{i j k}: B^{i j} \cdot B^{j k} \rightarrow B^{i k}$ denote the structure maps of $B$. 
The simplicial structure is as follows: if $\varphi:[q] \rightarrow[p] \in \Delta$ the functor $\varphi^{*}: B_{p}(\mathcal{C}) \rightarrow$ $B_{q}(\mathcal{C})$ is obtained by precomposing with $\varphi$. In order to allow for degeneracy maps $s_{i}$ we use the convention that all objects of the form $A^{i i}$ are the unit of the monoidal structure.

The $K$-theory of the bimonoidal category $\mathcal{R}$ can now be defined as usual. We take the bar constructions of the monoidal categories $\mathrm{GL}_{n} \mathcal{R}$ for all $n \geqslant 0$, realise them, take the disjoint union of all of these and group complete with respect to the block sum of matrices.

Definition 2.6 [4, Definition 3.12] For any bimonoidal category $\mathcal{R}$ its $K$-theory is

$$
\mathcal{K}(\mathcal{R})=\Omega B\left(\bigsqcup_{n \geqslant 0}\left|B \mathrm{GL}_{n} \mathcal{R}\right|\right)
$$

Note that $\mathcal{K}(\mathcal{R})$ is weakly equivalent to

$$
\mathbb{Z} \times|B \mathrm{GLR}|^{+} \text {. }
$$

Here, GL $\mathcal{R}$ is a suitable stabilization of the categories $\mathrm{GL}_{n} \mathcal{R}$ analogous to the stabilization of invertible matrices over a ring.

The main result of [3] states that $\mathcal{K}(\mathcal{R})$ is weakly equivalent to the algebraic $K$-theory of the ring spectrum associated to $\mathcal{R}, H \mathcal{R}$, if $\mathcal{R}$ is a small topological bimonoidal category satisfying the following conditions:

- All morphisms in $\mathcal{R}$ are isomorphisms.

- For every object $X \in \mathcal{R}$ the translation functor $X \oplus(-)$ is faithful.

\section{Bimonoidal categories with anti-involution}

In order to define an involution of $\mathcal{K}(\mathcal{R})$ we need to assume some extra structure on our bimonoidal category $\mathcal{R}$, namely the existence of an anti-involution on $\mathcal{R}$. David Barnes considers involutions on monoidal categories in Barnes [5, Section 7]. We have to incorporate the full bimonoidal structure, but some of our axioms below relate to his.

Definition 3.1 An anti-involution in a strict bimonoidal category $\mathcal{R}$ consists of a functor $\zeta: \mathcal{R} \rightarrow \mathcal{R}$ with $\zeta \circ \zeta=$ id and such that there are natural isomorphisms

$$
\mu_{A, B}: \zeta(A \otimes B) \rightarrow \zeta(B) \otimes \zeta(A)
$$

for all $A, B \in \mathcal{R}$. In addition, the functor $\zeta$ and the isomorphisms $\mu$ have to satisfy the following properties. 
(a) The functor $\zeta$ is strictly symmetric monoidal with respect to $\left(\mathcal{R}, \oplus, 0_{\mathcal{R}}, c_{\oplus}\right)$ $[15, \mathrm{XI} .2]$.

(b) The multiplicative unit $1_{\mathcal{R}}$ is fixed under $\zeta$, ie, $\zeta\left(1_{\mathcal{R}}\right)=1_{\mathcal{R}}$ and $\mu_{1_{\mathcal{R}}, A}=$ $\operatorname{id}_{\zeta(A)}=\mu_{A, 1_{\mathcal{R}}}$.

(c) The isomorphisms $\mu$ are associative in the sense that the diagram

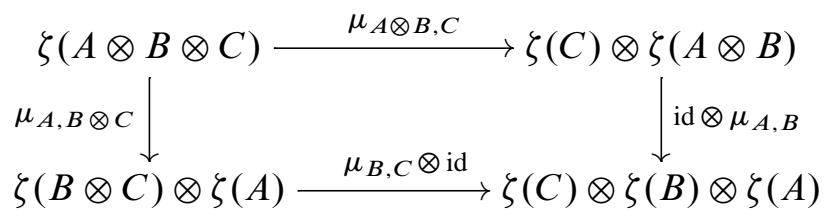

commutes for all $A, B, C \in \mathcal{R}$.

(d) The distributivity isomorphisms $d_{r}$ and $d_{\ell}$ and the isomorphisms $\mu$ render the following diagrams commutative:

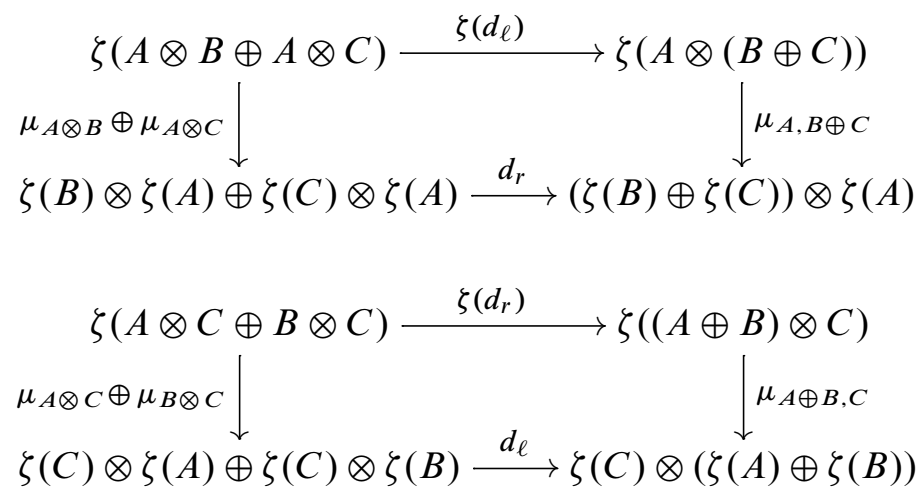

For a bimonoidal category with anti-involution $(\mathcal{R}, \zeta, \mu)$ the objects that are fixed under the anti-involution $\zeta$ do not form a bimonoidal category in general. They carry a permutative structure with respect to $\oplus$.

Remark 3.2 In the case of rings an anti-involution is a map from a ring $R$ to the ring $R^{o}$ where $R^{o}$ has the same additive structure as $R$ but has reversed multiplication. In a similar spirit one can define a bimonoidal category $\mathcal{R}^{o}$ for any bimonoidal category $\mathcal{R}$ where the multiplicative structure is reversed. However, the right distributivity in $\mathcal{R}^{o}$ is then no longer the identity because it corresponds to the left distributivity law in $\mathcal{R}$. An anti-involution for a bimonoidal category $\mathcal{R}$ can be viewed as a lax morphism of bimonoidal categories from $\mathcal{R}$ to $\mathcal{R}^{o}$ by adapting [2] to a setting with $d_{r} \neq \mathrm{id}$. 
Definition 3.3 A morphism $F:(\mathcal{R}, \zeta, \mu) \rightarrow\left(\mathcal{R}^{\prime}, \zeta^{\prime}, \mu^{\prime}\right)$ of bimonoidal categories with anti-involution is a lax bimonoidal functor $F: \mathcal{R} \rightarrow \mathcal{R}^{\prime}$ with the additional properties that

$$
F \circ \zeta=\zeta^{\prime} \circ F
$$

and that $\mu$ and $\mu^{\prime}$ are compatible with the transformations

$$
\lambda^{A, B}: F(A) \otimes F(B) \rightarrow F(A \otimes B)
$$

in the sense that the diagram

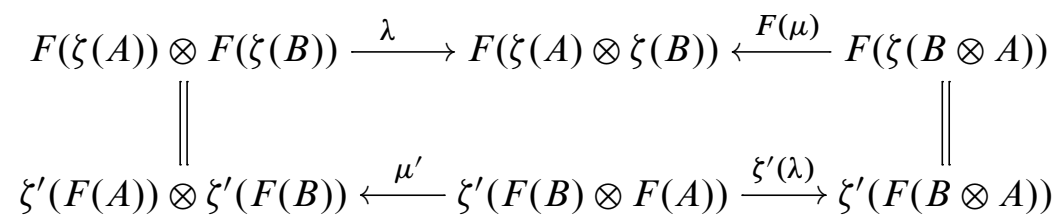

commutes for all $A, B$ in $\mathcal{R}$.

We extend the anti-involution $\zeta$ to the category of matrices $M_{n}(\mathcal{R})$ coordinatewise, so for any $A=\left(A_{i, j}\right)_{i, j} \in M_{n}(\mathcal{R})$

$$
\zeta\left(\left(A_{i, j}\right)_{i, j}\right)=\left(\zeta\left(A_{i, j}\right)\right)_{i, j} .
$$

If the matrix $A$ is an element in $\mathrm{GL}_{n}(\mathcal{R})$ then so is $\zeta(A)$, and $\zeta\left(E_{n}\right)=E_{n}$.

\section{The anti-involution on $\mathcal{K}(\mathcal{R})$}

Regardless of the special form of a bimonoidal category with anti-involution, $(\mathcal{R}, \zeta, \mu)$, the combinatorial nature of the bar construction $B \mathrm{GL}(\mathcal{R})$ allows for a canonical involution map.

In the following $\mathcal{R}$ is always a fixed bimonoidal category with anti-involution.

Definition 4.1 For a matrix of objects $A \in M_{n}(\mathcal{R})$ the transpose of $A, A^{t}$, has $A_{i, j}^{t}=A_{j, i}$ as entries. For a morphism $\phi: A \rightarrow C$ in $M_{n}(\mathcal{R})$ we define $\phi^{t}$ as

$$
\phi_{i, j}^{t}:=\phi_{j, i}: A_{j, i}=A_{i, j}^{t} \rightarrow C_{i, j}^{t}=C_{j, i}
$$


For a general bimonoidal category, the formula that we are used to, namely $(A \cdot B)^{t}=$ $B^{t} \cdot A^{t}$ does not hold on the nose, but only up to a twist. We have

whereas

$$
(A \cdot B)_{i, j}^{t}=(A \cdot B)_{j, i}=\bigoplus_{k=1}^{n} A_{j, k} \otimes B_{k, i}
$$

$$
\left(B^{t} \cdot A^{t}\right)_{i, j}=\bigoplus_{k=1}^{n} B_{i, k}^{t} \otimes A_{k, j}^{t}=\bigoplus_{k=1}^{n} B_{k, i} \otimes A_{j, k} .
$$

Using the structure maps $\mu$ of the anti-involution on $\mathcal{R}$, we define $\mu:(\zeta(A \cdot B))^{t} \rightarrow$ $\zeta(B)^{t} \cdot \zeta(A)^{t}$ by setting $\mu=\bigoplus_{k=1}^{n} \mu_{A_{j, k}, B_{k, i}}$. The map $\mu$ behaves well on morphisms.

Lemma 4.2 For morphisms $\phi: A \rightarrow C$ and $\psi: B \rightarrow D$ in $M_{n}(\mathcal{R})$ the following diagram commutes:

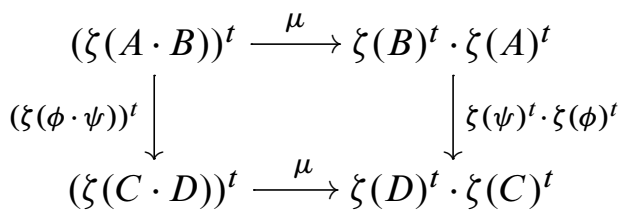

Proof The $(i, j)$ matrix component of the diagram above is

$$
\begin{gathered}
\bigoplus_{k=1}^{n} \zeta\left(A_{j, k} \otimes B_{k, i}\right) \stackrel{\oplus_{k} \mu_{A_{j, k}, B_{k, i}}}{\longrightarrow} \bigoplus_{k=1}^{n} \zeta\left(B_{k, i}\right) \otimes \zeta\left(A_{j, k}\right) \\
\bigoplus_{k} \zeta\left(\phi_{j, k} \otimes \psi_{k, i}\right) \downarrow \\
\bigoplus_{k=1}^{n} \zeta\left(C_{j, k} \otimes D_{k, i}\right) \stackrel{\oplus_{k} \mu_{C_{j, k}, D_{k, i}}}{\longrightarrow} \bigoplus_{k=1}^{n} \zeta\left(\psi_{k, i}\right) \otimes \zeta\left(\phi_{j, k}\right) \\
\left.\stackrel{D_{k, i}}{ }\right) \otimes \zeta\left(C_{j, k}\right)
\end{gathered}
$$

and this commutes because $\mu$ is natural.

\section{Definition 4.3 Let}

$$
\begin{array}{ccc}
A^{0,1} & \cdots & A^{0, q} \\
& \ddots & \vdots \\
& & A^{q-1, q}
\end{array}
$$

together with coherent isomorphisms $\phi^{i, j, k}: A^{i, j} \cdot A^{j, k} \rightarrow A^{i, k}, 0 \leqslant i<j<k \leqslant q$, be an element in $B_{q} \mathrm{GL}_{n}(\mathcal{R})$. 
We define $\tau: B_{q} \mathrm{GL}_{n}(\mathcal{R}) \rightarrow B_{q} \mathrm{GL}_{n}(\mathcal{R})$ via

$$
\begin{aligned}
& A^{0,1} \ldots A^{0, q} \quad\left(\zeta\left(A^{q-1, q}\right)\right)^{t} \ldots\left(\zeta\left(A^{0, q}\right)\right)^{t} \\
& \tau: \\
& \ddots \underset{A^{q-1, q}}{\vdots} \mapsto \\
& \cdot \begin{array}{c}
\ddots \\
\left(\zeta\left(A^{0,1}\right)\right)^{t} .
\end{array}
\end{aligned}
$$

Let $B^{i, j}$ denote $\left(\zeta\left(A^{q-j, q-i}\right)\right)^{t}$. The corresponding isomorphisms

$$
\tau(\phi)^{i, j, k}: B^{i, j} \cdot B^{j, k} \rightarrow B^{i, k}
$$

for $0 \leqslant i<j<k \leqslant q$ are given by

$$
\begin{gathered}
\left.\tau(\phi)^{i, j, k}: \zeta\left(A^{q-j, q-i}\right)\right)^{t} \cdot\left(\zeta\left(A^{q-k, q-j}\right)\right)^{t} \\
\qquad \mu^{-1} \\
\quad\left(\zeta\left(A^{q-k, q-j} \cdot A^{q-j, q-i}\right)\right)^{t} \stackrel{\left(\zeta\left(\phi^{q-k, q-j, q-i}\right)\right)^{t}}{\longrightarrow}\left(\zeta\left(A^{q-k, q-i}\right)\right)^{t} .
\end{gathered}
$$

Let $\alpha=\alpha_{A, B, C}: A \cdot(B \cdot C) \longrightarrow(A \cdot B) \cdot C$ be the natural associativity isomorphism in the monoidal structure of $\left(\mathrm{GL}_{n} \mathcal{R}, \cdot, E_{n}\right)$. We can express $\alpha$ in terms of distributivity maps and additive twist maps as follows: let $\sigma=\left(\sigma_{i, j}\right)$ be the additive twist

$$
\sigma_{i, j}: \bigoplus_{k=1}^{n} \bigoplus_{\ell=1}^{n} A_{i, k} \otimes B_{k, \ell} \otimes C_{\ell, j} \longrightarrow \bigoplus_{\ell=1}^{n} \bigoplus_{k=1}^{n} A_{i, k} \otimes B_{k, \ell} \otimes C_{\ell, j}
$$

that exchanges the priority of summation of the two sums. Then

$$
\alpha=d_{r} \circ \sigma \circ d_{\ell}^{-1}=\sigma \circ d_{\ell}^{-1} .
$$

Here, the distributivity law is applied to sums of $n$ entries. This does not cause problems as addition is assumed to be strictly associative. The fact that $\alpha$ satisfies Mac Lane's pentagon axiom [15, VII.1(5)] can be seen by brute-force comparison of terms using the axioms [11, Definition 3.3].

Lemma 4.4 The associativity isomorphism for matrix multiplication, $\alpha$, and the isomorphisms $\mu$ are compatible, ie, they satisfy

$$
(\mathrm{id} \cdot \mu) \circ \mu \circ \zeta(\alpha)^{t}=\alpha^{-1} \circ(\mu \cdot \mathrm{id}) \circ \mu .
$$

Proof To ease notation, we will abbreviate $A \otimes B$ to $A B$. The $(i, j)$ matrix component of the equation

$$
(\mathrm{id} \cdot \mu) \circ \mu \circ \zeta(\alpha)^{t}=\alpha^{-1} \circ(\mu \cdot \mathrm{id}) \circ \mu: \zeta(A \cdot(B \cdot C))^{t} \longrightarrow \zeta(C)^{t} \cdot\left(\zeta(B)^{t} \cdot \zeta(A)^{t}\right)
$$


that we want to have is part of the diagram:

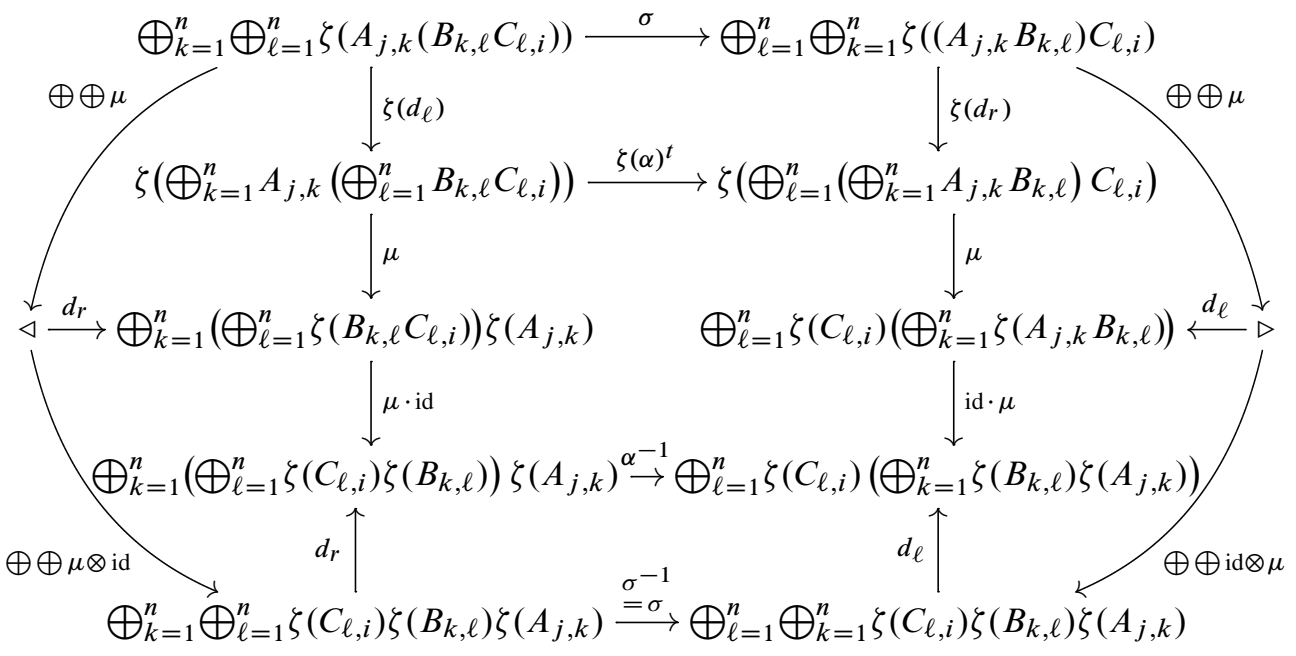

Here, the symbol $\triangleleft$ on the left hand side stands for $\bigoplus_{k=1}^{n} \bigoplus_{\ell=1}^{n} \zeta\left(B_{k, \ell} C_{\ell, i}\right) \zeta\left(A_{j, k}\right)$ and the $\triangleright$ on the right hand side is short for $\bigoplus_{\ell=1}^{n} \bigoplus_{k=1}^{n} \zeta\left(C_{\ell, i}\right) \zeta\left(A_{j, k} B_{k, \ell}\right)$. From the definition of an anti-involution we know that the top triangles and the outer diagram commute. Naturality of the distributivity transformations makes the bottom triangles commute and therefore the square in the middle commutes as well.

Lemma 4.5 The isomorphisms $\tau(\phi)^{i, j, k}$ as in (3) are coherent.

Proof Recall that the $\phi^{i, j, k}$ are the coherence isomorphisms for the triangle of matrices $\left(A^{i, j}\right)_{i, j}$ and that $B^{i j}=\left(\zeta\left(A^{q-j, q-i}\right)\right)^{t}$. We have to prove that the following diagram commutes.

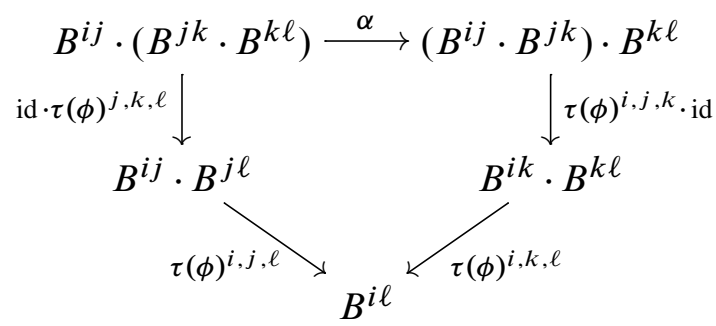

As $\tau(\phi)^{j, k, \ell}$ is the composition $\left(\zeta\left(\phi^{q-\ell, q-k, q-j}\right)\right)^{t} \circ \mu^{-1}$, as we know from naturality of $\mu$ that

$$
\begin{aligned}
\mu^{-1} \circ\left(\left(\zeta\left(\phi^{q-k, q-j, q-i}\right)\right)^{t} \cdot \mathrm{id}\right) & =\mu^{-1} \circ\left(\left(\zeta\left(\phi^{q-k, q-j, q-i}\right)\right)^{t} \cdot \mathrm{id}^{t}\right) \\
& =\left(\mathrm{id} \cdot \zeta\left(\phi^{q-k, q-j, q-i}\right)\right)^{t} \circ \mu^{-1}
\end{aligned}
$$


and as we have Lemma 4.4, it suffices to show that the diagram

$$
\begin{aligned}
& \zeta\left(A^{q-\ell, q-k} \cdot\left(A^{q-k, q-j} \cdot A^{q-j, q-i}\right)\right)^{t} \stackrel{\zeta(\alpha)^{t}}{\longrightarrow} \zeta\left(\left(A^{q-\ell, q-k} \cdot A^{q-k, q-j}\right) \cdot A^{q-j, q-i}\right)^{t} \\
& \zeta\left(\mathrm{id} \cdot\left(\phi^{q-k, q-j, q-i}\right)\right)^{t} \downarrow \quad \downarrow\left(\zeta\left(\phi^{q-\ell, q-k, q-j}\right) \cdot \mathrm{id}\right)^{t} \\
& \zeta\left(A^{q-\ell, q-k} \cdot A^{q-k, q-i}\right)^{t} \quad \zeta\left(A^{q-\ell, q-j} \cdot A^{q-j, q-i}\right)^{t} \\
& \zeta\left(\phi^{q-\ell, q-k, q-i}\right)^{t} \underbrace{}_{\zeta\left(A^{q-\ell, q-i}\right)^{t}} \overbrace{\zeta\left(\phi^{q-\ell, q-j, q-i}\right)^{t}}
\end{aligned}
$$

commutes. As both transposition and $\zeta$ are functors, the commutativity of this diagram is equivalent to the equality

$$
\phi^{q-\ell, q-j, q-i} \circ\left(\phi^{q-\ell, q-k, q-j} \cdot \mathrm{id}\right) \circ \alpha=\phi^{q-\ell, q-k, q-i} \circ\left(\mathrm{id} \cdot \phi^{q-k, q-j, q-i}\right)
$$

and this holds because the isomorphisms $\left(\phi^{q-\ell, q-j, q-i}\right)$ are coherent.

Remark 4.6 If $G$ is a group, then the inverse map induces a map on the level of classifying spaces $B \iota: B G \rightarrow B G^{\mathrm{op}}$. Here, $G^{\mathrm{op}}$ is the group $G$ with opposite multiplication. This map is homotopic to the map $\kappa: B G \rightarrow B G^{\text {op }}$ which sends $\left(\left(g_{1}, \ldots, g_{q}\right),\left(t_{0}, \ldots, t_{q}\right)\right) \in B_{q} G$ to $\left(\left(g_{q}, \ldots, g_{1}\right),\left(t_{q}, \ldots, t_{0}\right)\right) \in B G^{\text {op }}$ (see Burghelea and Fiedorowicz [7, page 206] for an explicit homotopy). Note that $\kappa$ can be defined for small strict monoidal categories as well.

Let $r: \Delta^{\mathrm{op}} \rightarrow \Delta^{\mathrm{op}}[19,(3.14)]$ be the following functor: on objects $r$ is the identity. If $f=g^{\text {op }}:[p] \rightarrow[q]$ is a morphism in $\Delta^{\text {op }}$ then $r(f)$ is the opposite of the monotone map that is given by

$$
i \mapsto p-g(q-i), \quad \text { for all } \quad 0 \leqslant i \leqslant q .
$$

Let CAT denote the category of small categories.

Lemma 4.7 If $\widetilde{B} \mathrm{GL}_{n}(\mathcal{R})$ denotes the bar construction of $\mathrm{GL}_{n} \mathcal{R}$ with respect to the simplicial structure

$$
\Delta^{\mathrm{op}} \stackrel{r}{\longrightarrow} \Delta^{\mathrm{op}} \stackrel{B \mathrm{GL}_{n} \mathcal{R}}{\longrightarrow} \mathrm{CAT},
$$

then $\tau$ induces a well-defined map of simplicial categories

$$
\tau: B \mathrm{GL}_{n} \mathcal{R} \rightarrow \widetilde{B} \mathrm{GL}_{n} \mathcal{R}
$$

for all $n$. 
Proof We use the following abbreviation: for $p \geqslant 0$ let $\omega_{p}:[p] \rightarrow[p]$ be the map, that sends $i$ to $p-i$. Then for any $f=g^{\text {op: }}[p] \rightarrow[q]$ we have that $r(f)$ is the opposite of the map $\omega_{p} \circ g \circ \omega_{q}$. Let $0 \leqslant i<j<k \leqslant p$ and $0 \leqslant k<\ell<m \leqslant q$. For an object $\left(A^{i, j}\right)$ in $B_{p}\left(\mathrm{GL}_{n}(\mathcal{R})\right)$ with coherence isomorphisms $\left(\phi^{i, j, k}\right)$ the involution maps the pair $\left(A^{i, j}\right)_{i, j}$ to $\left(\zeta\left(A^{\omega_{p}(j), \omega_{p}(i)}\right)^{t}\right)_{i, j}$ and the map $r(f)$ induces

$$
\left(\omega_{p} \circ g \circ \omega_{q}\right)^{\mathrm{op}}\left(\zeta\left(A^{\omega_{p}(j), \omega_{p}(i)}\right)^{t}\right)_{i, j}=\left(\zeta\left(A^{\omega_{p}^{2}\left(g\left(\omega_{q}(\ell)\right)\right), \omega_{p}^{2}\left(g\left(\omega_{q}(k)\right)\right)}\right)^{t}\right)_{k, \ell} .
$$

First applying $g^{\text {op }}$ and then using the involution $\tau$ amounts to the composition

$$
\left(A^{i, j}\right)_{i, j} \mapsto\left(A^{g(i), g(j)}\right)_{i, j} \mapsto\left(\zeta\left(A^{g\left(\omega_{q}(\ell)\right), g\left(\omega_{q}(k)\right)}\right)^{t}\right)_{k, \ell} .
$$

As $\omega_{p}^{2}$ is the identity, the two terms agree.

For the coherence condition the composition $\tau \circ f$ gives

$$
\left(\phi^{i, j, k}\right)_{i, j, k} \mapsto\left(\phi^{g(i), g(j), g(k)}\right)_{i, j, k} \mapsto\left(\zeta\left(\phi^{g\left(\omega_{q}(m)\right), g\left(\omega_{q}(\ell)\right), g\left(\omega_{q}(k)\right)}\right)^{t} \circ \mu^{-1}\right)_{k, \ell, m}
$$

whereas $r(f) \circ \tau$ induces

$$
\begin{aligned}
& \left(\phi^{i, j, k}\right)_{i, j, k} \longmapsto\left(\zeta\left(\phi^{\omega_{q}(m), \omega_{q}(\ell), \omega_{q}(k)}\right)^{t} \circ \mu^{-1}\right)_{k, \ell, m} \\
& \downarrow \\
& \left(\zeta\left(\phi^{\omega_{p}^{2} \circ g\left(\omega_{q}(m)\right), \omega_{p}^{2} \circ g\left(\omega_{q}(\ell)\right), \omega_{p}^{2} \circ g\left(\omega_{q}(k)\right)}\right)^{t} \circ \mu^{-1}\right)_{k, \ell, m}
\end{aligned}
$$

and this coincides with the above term.

Theorem 4.8 The involution $\tau$ gives rise to an involution $\tilde{\tau}$ on $\mathcal{K}(\mathcal{R})$ for every bimonoidal category with anti-involution $(\mathcal{R}, \zeta, \mu)$.

Proof We saw that the involution $\tau$ is a morphism of simplicial categories

$$
\tau: B \mathrm{GL}_{n} \mathcal{R} \rightarrow \widetilde{B} \mathrm{GL}_{n} \mathcal{R}
$$

thus it remains to show that the realization of $\widetilde{B} \mathrm{GL}_{n} \mathcal{R},\left|\widetilde{B} \mathrm{GL}_{n} \mathcal{R}\right|$ is homeomorphic to $\left|B \mathrm{GL}_{n} \mathcal{R}\right|$ and that the involution passes to the group completion.

Let $\Upsilon:\left|\widetilde{B} \mathrm{GL}_{n} \mathcal{R}\right| \rightarrow\left|B \mathrm{GL}_{n} \mathcal{R}\right|$ denote the map on the geometric realization that is defined as

$$
\Upsilon\left[\begin{array}{ccc}
A^{0,1} & \cdots & A^{0, q} \\
& \ddots & \vdots \\
& & A^{q-1, q}
\end{array},\left(t_{0}, \ldots, t_{q}\right)\right]=\left[\begin{array}{ccc}
A^{0,1} & \cdots & A^{0, q} \\
& \ddots & \vdots \\
& & A^{q-1, q}
\end{array},\left(t_{q}, \ldots, t_{0}\right)\right] .
$$


If $\delta_{i}:[q-1] \rightarrow[q]$ denotes the map that is the inclusion that misses $i$ and is strictly monotone everywhere else and if $\sigma_{i}:[q+1] \rightarrow[q]$ is the surjection that sends $i$ and $i+1$ to $i$ and is strictly monotone elsewhere, then note that $\delta_{q-i} \circ \omega_{q-1}=\omega_{q} \circ \delta_{i}$ and $\sigma_{q-i} \circ \omega_{q+1}=\omega_{q} \circ \sigma_{i}$. These identities ensure that $r\left(d_{i}\right)=d_{q-i}, r\left(s_{i}\right)=s_{q-i}$ for $d_{i}=\left(\delta_{i}\right)^{\mathrm{op}}, s_{i}=\left(\sigma_{i}\right)^{\mathrm{op}}$ and that $\Upsilon$ is well-defined.

We define $\tilde{\tau}$ as $\Upsilon \circ \tau:\left|B \mathrm{GL}_{n} \mathcal{R}\right| \rightarrow\left|B \mathrm{GL}_{n} \mathcal{R}\right|$. The composition $\tilde{\tau} \circ \tilde{\tau}$ is the identity on $\left|B \mathrm{GL}_{n} \mathcal{R}\right|$ because the map $\tau$ commutes with $\Upsilon$ and both maps are involutions. As $\mathcal{K}(\mathcal{R})=\Omega B\left(\bigsqcup_{n \geqslant 0}\left|B \mathrm{GL}_{n} \mathcal{R}\right|\right)$, we have to show that $\tilde{\tau}$ is compatible with the monoid structure on $\bigsqcup_{n \geqslant 0}\left|B \mathrm{GL}_{n} \mathcal{R}\right|$. Note that the following diagram commutes



and we obtain on the level of classifying spaces that

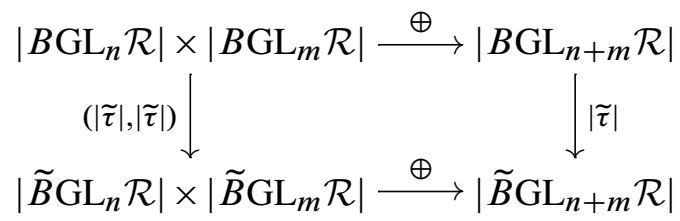

commutes as well.

Proposition 4.9 If $F:(\mathcal{R}, \zeta, \mu) \rightarrow\left(\mathcal{R}^{\prime}, \zeta^{\prime}, \mu^{\prime}\right)$ is a morphism of bimonoidal categories with anti-involution, then $F$ commutes with the involutions on $\mathcal{K} \mathcal{R}$ and $\mathcal{K} \mathcal{R}^{\prime}$, ie, $F \circ \tilde{\tau}=\tilde{\tau} \circ F$,

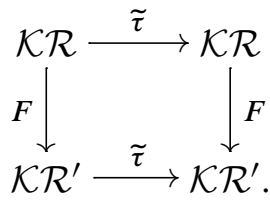

Let $R$ be an associative ring with unit. An anti-involution on $R$ (called involution in [7, Definition 1.1]) is a function $\iota: R \rightarrow R$ with $\iota(\iota(a))=a, \iota(a+b)=\iota(a)+\iota(b)$ and $\iota(a b)=\iota(b) \iota(a)$ for all $a, b \in R$.

Definition 4.10 If $R$ is a ring or a rig, then the category which has the elements of $R$ as objects and only identity morphisms is a bimonoidal category. We denote this category by $\mathcal{R}_{R}$ and call it the discrete category associated to the ring or rig $R$. If $R$ is commutative, then $\mathcal{R}_{R}$ is bipermutative. 
If $R$ is a ring then the spectrum associated to $\mathcal{R}_{R}$ is the Eilenberg-Mac Lane spectrum of the ring $R$. For a rig $R$, we obtain the Eilenberg-MacLane spectrum of the group completion $\operatorname{Gr}(R)$.

Note that for a small bimonoidal category with anti-involution $(\mathcal{R}, \zeta, \mu)$, the set of path components $\pi_{0}(\mathcal{R})$, is a rig with anti-involution.

Corollary 4.11 For a small bimonoidal category with anti-involution $\left(\mathcal{R}^{\prime}, \zeta, \mu\right)$, the map $\mathcal{K}\left(\mathcal{R}^{\prime}\right) \rightarrow \mathcal{K}\left(\mathcal{R}_{\pi_{0}\left(\mathcal{R}^{\prime}\right)}\right)$ commutes with the involutions on $\mathcal{K}\left(\mathcal{R}^{\prime}\right)$ and $\mathcal{K}\left(\mathcal{R}_{\pi_{0}\left(\mathcal{R}^{\prime}\right)}\right) \simeq$ $K^{f}\left(\operatorname{Gr}\left(\pi_{0}\left(\mathcal{R}^{\prime}\right)\right)\right)$.

Proposition 4.12 For a ring with anti-involution the involution constructed on $\mathcal{K}\left(\mathcal{R}_{R}\right)$ agrees with the standard involution on $K_{i}(R), i \geqslant 1$.

Proof As GL $\mathcal{R}_{R}$ is a strict monoidal category, the bar construction from Section 2 is equivalent to the ordinary bar construction [3] and the isomorphism from the ordinary bar construction to the one in the monoidal setting is given by sending a $q$-simplex of the ordinary bar construction $\left(B_{0}, \ldots, B_{q}\right)$ to the triangle in $B_{q} \mathrm{GL}_{n} \mathcal{R}_{R}$

$$
\begin{array}{ccc}
A^{0,1} & \cdots & A^{0, q} \\
& \ddots & \vdots \\
& & A^{q-1, q}
\end{array}
$$

with entries $A^{i, i+1}=B_{i}$ on the diagonal. The other entries are given by iterated matrix multiplication of the $B_{i}$ s and the isomorphisms $\phi^{i j k}$ are chosen to be identity maps. On the diagonal the involution $\tau$ sends $\left(B_{0}, \ldots, B_{q}\right)$ to $\left(\zeta\left(B_{q}\right)^{t}, \ldots, \zeta\left(B_{0}\right)^{t}\right)$ and this is precisely what the standard involution in algebraic $K$-theory does (compare for instance [7, Definition 1.12]).

Note that if one is willing to work away from the prime 2, then involutions give rise to splittings

$$
\mathcal{K}(\mathcal{R}) \sim \mathcal{K}(\mathcal{R})^{a} \times \mathcal{K}(\mathcal{R})^{s}
$$

of $\mathcal{K}(\mathcal{R})$ into an antisymmetric part, $\mathcal{K}(\mathcal{R})^{a}$, and a symmetric part, $\mathcal{K}(\mathcal{R})^{s}$. Corollary 4.11 tells us that such splittings are compatible with the path component map.

Remark 4.13 There is no straightforward way to mimic Burghelea's and Fiedorowicz's construction of hermitian $K$-theory in the setting of bimonoidal categories with antiinvolution. There are two main obstacles: matrix multiplication is not associative any longer and we do not demand that the structure isomorphism $\mu$ is the identity. This 
has the effect that the analogue of their category ${ }_{\varepsilon} O_{n}[7,1.2]$ in the bimonoidal world does not give a strict category.

Similarly, their bar construction description of ${ }_{\varepsilon} O_{n}$ does not have a direct analogue. In order to form the one-sided bar construction $B_{1}\left(\operatorname{Sym}_{n}^{1}(\mathcal{R}), \mathrm{GL}_{n}(\mathcal{R}), *\right)$ in the spirit of $[7,1.3]$ one has to have an action of the monoidal category $\operatorname{GL}_{n}(\mathcal{R})$ on the category of symmetric matrices $\operatorname{Sym}_{n}^{1}(\mathcal{R})$. Here, the objects of $\operatorname{Sym}_{n}^{1}(\mathcal{R})$ are matrices $A \in \mathrm{GL}_{n}(\mathcal{R})$ with $\zeta(A)^{t}=A$ and morphisms are morphisms in $\mathrm{GL}_{n}(\mathcal{R})$ that are untouched by $\zeta$. But for $M \in \operatorname{Sym}_{n}^{1}(\mathcal{R})$ and $A \in \operatorname{GL}_{n}(\mathcal{R})$ the object $\left(\zeta(A)^{t} \cdot M\right) \cdot A$ will only be symmetric up to isomorphism in general.

The involution on hermitian $K$-theory [7, 4.1] is induced by the map that sends a symmetric matrix $A$ to its negative. We know from [3] that $\mathcal{K}(\mathcal{R})$ is equivalent to $\mathcal{K}(\overline{\mathcal{R}})$ for some multiplicative group completion $\overline{\mathcal{R}}$ of $\mathcal{R}$ and matrices over $\overline{\mathcal{R}}$ have additive inverses on the level of path components.

We can produce other examples of bimonoidal categories with anti-involution along the following lines.

Let $\mathcal{E}$ denote the bipermutative category of finite sets whose objects are the finite sets $\mathbf{n}=\{1, \ldots, n\}$ for $n \in \mathbb{N}_{0}$. By convention $\mathbf{0}$ is the empty set. The morphisms in $\mathcal{E}$ are

$$
\mathcal{E}(\mathbf{n}, \mathbf{m})= \begin{cases}\Sigma_{n} & n=m, g=h \text { or } n=m=0, \\ \varnothing & \text { otherwise. }\end{cases}
$$

For the full structure see May [16, VI, Example 5.1] or Baas et al [2].

For a discrete group $G$ let $\vee_{G} \mathcal{E}$ be the category with objects $\mathbf{n}_{g}$ with $n \in \mathbb{N}_{0}$ and $g \in G$. We identify all objects $\mathbf{0}_{g}$ to $\mathbf{0}$ which stands for the empty set and the $\mathbf{n}_{g}$ should be thought of as the set $\{1, \ldots, n\}$ labelled by $g \in G$. Morphisms are given by

$$
\vee_{G} \mathcal{E}\left(\mathbf{n}_{g}, \mathbf{m}_{h}\right)= \begin{cases}\varnothing & n \neq m, \\ \Sigma_{n} & n=m, g=h \text { or } n=m=0 .\end{cases}
$$

The classifying space of $\vee_{G} \mathcal{E}$ is

$$
B\left(\vee_{G} \mathcal{E}\right)=\bigvee_{G} B(\mathcal{E})=\bigvee_{G}\left(\bigsqcup_{n \geqslant 0} B \Sigma_{n}\right)
$$

We define a bimonoidal structure on $\vee_{G} \mathcal{E}$ as follows. Objects can only be added if their indices agree:

$$
\mathbf{n}_{g} \oplus \mathbf{m}_{h}= \begin{cases}(\mathbf{n}+\mathbf{m})_{g} & g=h, \\ \mathbf{0} & g \neq h\end{cases}
$$


and we define the multiplication to be $\mathbf{n}_{g} \otimes \mathbf{m}_{h}=(\mathbf{n} \otimes \mathbf{m})_{g h}$. The additive twist, $c_{\oplus}$ on $\mathcal{E}_{G}$, is inherited from $\mathcal{E}, \mathbf{0}$ is the zero object and $\mathbf{1}_{e}$ is the multiplicative unit, if $e$ denotes the neutral element of the group $G$.

With this structure $\vee_{G} \mathcal{E}$ is a bimonoidal category; if $G$ is abelian, then $\vee_{G} \mathcal{E}$ is actually bipermutative.

We can define an anti-involution on $\vee_{G} \mathcal{E}$ for any discrete $G$ via

$$
\zeta\left(\mathbf{n}_{g}\right)=\mathbf{n}_{g-1} .
$$

Note that the isomorphisms $\mu$ are not trivial in this case, but

$$
\begin{aligned}
\zeta\left(\mathbf{n}_{g} \otimes \mathbf{m}_{h}\right) & =\zeta\left((\mathbf{n} \otimes \mathbf{m})_{g h}\right)=(\mathbf{n} \otimes \mathbf{m})_{(g h)^{-1}}=(\mathbf{n} \otimes \mathbf{m})_{h^{-1} g^{-1}} \\
& \neq \zeta\left(\mathbf{m}_{h}\right) \otimes \zeta\left(\mathbf{n}_{g}\right)=(\mathbf{m} \otimes \mathbf{n})_{h^{-1} g^{-1}}
\end{aligned}
$$

so we define $\mu$ to be $c_{\otimes}$ where $c_{\otimes}$ is the multiplicative twist in the bipermutative structure of $\mathcal{E}$. We have that $\zeta\left(1_{e}\right)=1_{e}$ and condition (7) follows from the equation

$$
d_{\ell} \circ\left(c_{\otimes} \oplus c_{\otimes}\right)=c_{\otimes} \circ d_{r}
$$

in bipermutative categories and the associativity of $\mu$ is a consequence of Lemma 5.3. The path components of $\vee_{G} \mathcal{E}$ constitute the monoid ring $\mathbb{N}_{0}[G]$ and therefore we obtain with Corollary 4.11 that the induced map on $K$-theory

$$
\mathcal{K}\left(\vee_{G} \mathcal{E}\right) \rightarrow \mathcal{K}\left(\mathbb{N}_{0}[G]\right)
$$

is compatible with the involutions on both sides. Note that $\mathcal{K}\left(\mathbb{N}_{0}[G]\right) \simeq \mathcal{K}(\mathbb{Z}[G])$.

\section{Braided bimonoidal and bipermutative categories}

We will show that braided bimonoidal, and therefore in particular bipermutative categories, provide examples of bimonoidal categories with anti-involution.

Definition 5.1 A braided bimonoidal category $\left(\mathcal{R}, \oplus, 0_{\mathcal{R}}, c_{\oplus}, \otimes, 1_{\mathcal{R}}, \beta\right)$ consists of a permutative category $\left(\mathcal{R}, \oplus, 0_{\mathcal{R}}, c_{\oplus}\right)$ together with a strict braided monoidal category $\left(\mathcal{R}, \otimes, 1_{\mathcal{R}}, \beta\right)$ (see Mac Lane [15, XI.1]) where $\beta$ is the braiding

$$
\beta=\beta^{A, B}: A \otimes B \longrightarrow B \otimes A \text {. }
$$

These two structures interact via distributivity laws. We assume that the right distributivity isomorphism

$$
d_{r}: A \otimes B \oplus A^{\prime} \otimes B \longrightarrow\left(A \oplus A^{\prime}\right) \otimes B
$$


is the identity and that the left distributivity isomorphism is given in terms of $d_{r}$ and $\beta$, such that the following diagram commutes:

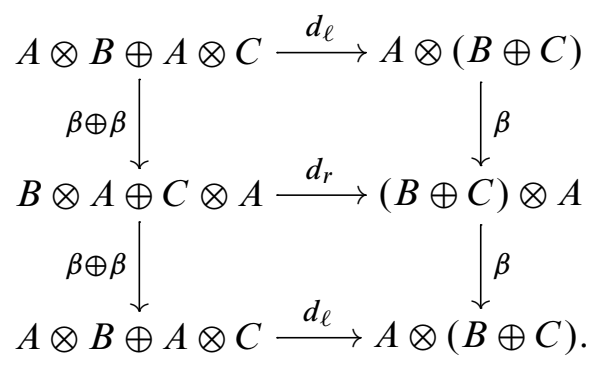

In addition we want that $\mathcal{R}$ satisfies the remaining axioms of a bipermutative category in the sense of [11, Definition 3.6].

Note that condition (7) implies that $\beta \circ \beta \circ d_{r}=d_{r} \circ(\beta \oplus \beta) \circ(\beta \oplus \beta)$ is satisfied.

Gerald Dunn studied braided bimonoidal categories and the reader might want to compare the above definition with [10, Definition 3.1]. As a class of examples of braided bimonoidal categories Dunn considered the category of what he called free crossed $G$-sets for a discrete group $G$ [9, example 2.3].

For every permutative category $\left(\mathcal{C}, \oplus, 0_{\mathcal{C}}, c_{\oplus}\right)$ one can construct the free braided bimonoidal category $\operatorname{Br}(\mathcal{C})$ along the lines of the construction in [11, Theorem 10.1]. Consider the translation category $E B r_{n}$ of the $n$-th braid group $B r_{n}$. Then

$$
\operatorname{Br}(\mathcal{C}):=\bigsqcup_{n \geqslant 0} E B r_{n} \times{ }_{B r_{n}} \mathcal{C}^{n}
$$

is a braided bimonoidal category (see Dunn [10, Proposition 3.5]). We present a different class of examples in Section 7.2.

In order to check that braided bimonoidal categories actually are bimonoidal categories with anti-involution and that they fit in the setting of our definition of $\mathcal{K}(\mathcal{R})$ in Section 2 we will need two technical results.

Lemma 5.2 Property (7) implies that the following diagram commutes:

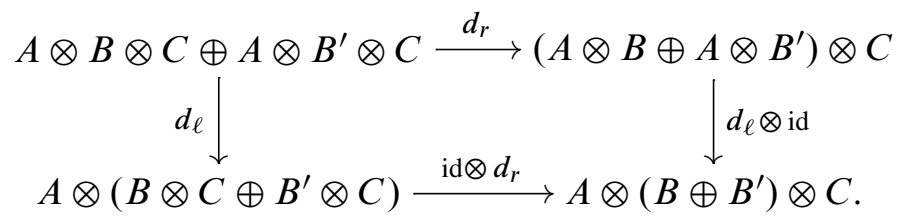


Proof We embed diagram (8) into the following diagram. In order to save space we use $A B$ for $A \otimes B$ and $A+B$ for $A \oplus B$.

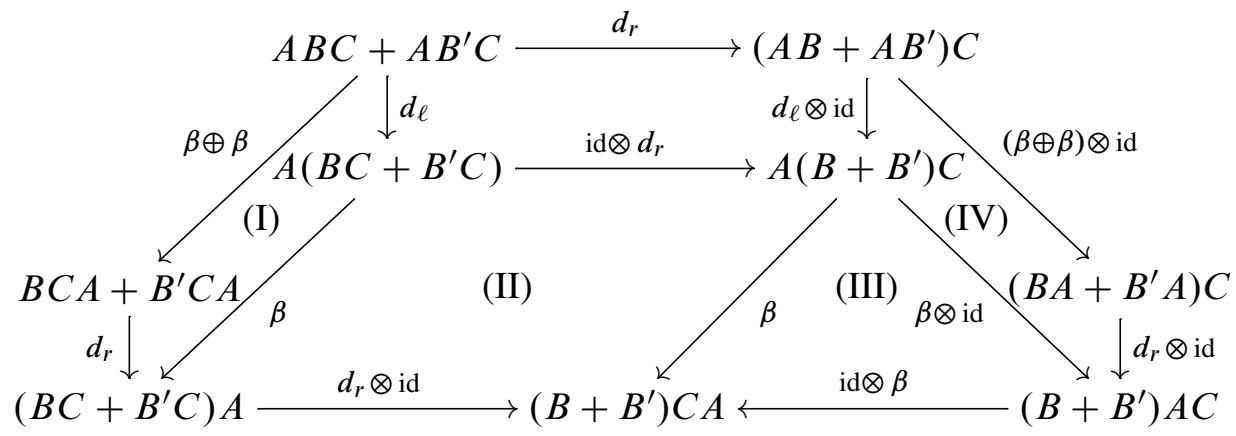

The leftmost subdiagram (I) corresponds precisely to property (7). Diagram (II) commutes because $\beta$ is natural and diagram (III) displays one of the axioms for a braided monoidal category and subdiagram (IV) again corresponds to property (7). As the right distributivity maps are identities, the outer diagram again corresponds to the property used in (III). Therefore the embedded subdiagram (8) commutes as well.

This result ensures that the axioms used in [2] are satisfied in the setting of braided bimonoidal categories. The next result is the key ingredient that allows us to interpret braided bimonoidal categories as bimonoidal categories with anti-involution.

Lemma 5.3 Let $\mathcal{R}$ be a braided bimonoidal category. Then the braiding $\beta$ satisfies

$$
\left(\mathrm{id} \otimes \beta^{A, B}\right) \circ \beta^{A \otimes B, C}=\left(\beta^{B, C} \otimes \mathrm{id}\right) \circ \beta^{A, B \otimes C} .
$$

Proof Consider the following diagram.

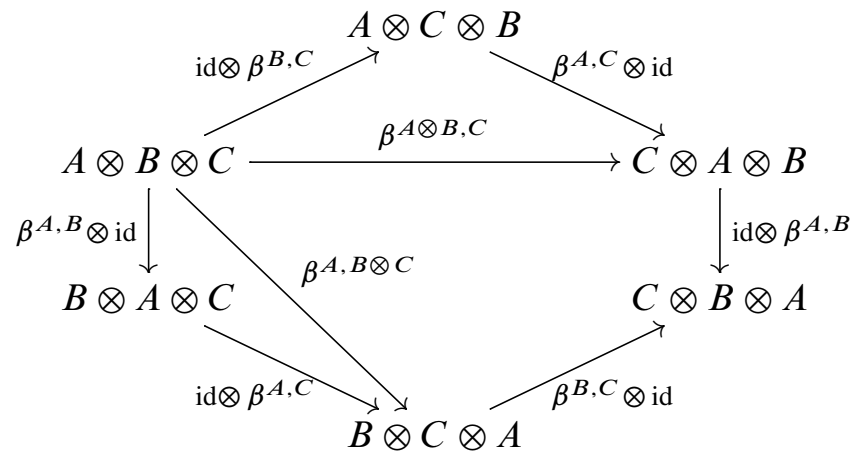

The two triangles display a coherence relation for braided monoidal categories and thus they commute. The outer diagram is the Yang-Baxter equation for the braiding and thus the whole diagram is commutative. 
Proposition 5.4 Every braided bimonoidal category is a bimonoidal category with anti-involution if one defines $\zeta$ to be the identity and $\mu=\beta$. In particular, every bipermutative category is a bimonoidal category with anti-involution with $\zeta=\mathrm{id}$ and $\mu=c_{\otimes}$.

Proof The claim follows directly from Lemma 5.3, because all other parts of the structure of a bimonoidal category with anti-involution are trivial.

Note that a morphism of bimonoidal categories with anti-involution as in Definition 3.3 specializes to the requirement of being a lax symmetric bimonoidal functor in the case of bipermutative categories.

\section{Group actions}

Let $G$ be a discrete group.

Definition 6.1 (a) Let $\mathcal{R}$ be a bimonoidal category and let $G$ be a discrete group. A $G$-action on $\mathcal{R}$ consists of a functor $\phi_{g}: \mathcal{R} \rightarrow \mathcal{R}$ for every $g \in G$, such that every $\phi_{g}$ is a strict bimonoidal functor and

$$
\phi_{1}=\mathrm{id}, \phi_{g} \circ \phi_{h}=\phi_{g h}, \text { for all } g, h \in G \text {. }
$$

(b) For a bimonoidal category with anti-involution we require each $\phi_{g}$ in addition to be a morphism of bimonoidal categories with anti-involution according to Definition 3.3.

Example 6.2 The bipermutative category of complex vector spaces, $\mathcal{V}_{\mathbb{C}}$, with objects the natural numbers with zero and morphisms

$$
\mathcal{V}_{\mathbb{C}}(n, m)= \begin{cases}\varnothing & n \neq m, \\ U(n) & n=m\end{cases}
$$

carries a $\mathbb{Z} / 2 \mathbb{Z}$-action. On objects the action is trivial, and on morphisms it is given by complex conjugation of unitary matrices. Note that the action is nontrivial on the endomorphisms $U(1)$ of the multiplicative unit.

Example 6.3 Let $A \rightarrow B$ be a $G$-Galois extension of commutative rings in the sense of [8]. We can consider the discrete bipermutative categories $\mathcal{R}_{A}$ and $\mathcal{R}_{B}$ as in Definition 4.10 . Then $\mathcal{R}_{B}$ is a bipermutative category with $G$-action. 
Definition 6.4 For a bimonoidal category $\mathcal{R}$ with $G$-action, the $G$-fixed category is the subcategory of $\mathcal{R}$ containing all objects and morphisms that are fixed under every $\phi_{g}, g \in G$. We denote this category by $\mathcal{R}^{G}$.

The following result is straightforward to see.

Lemma 6.5 The $G$-fixed category of a strict $G$-action on a bimonoidal category (with anti-involution) is again a bimonoidal category (with anti-involution).

Example 6.6 If $R$ is a ring with a $G$-action, then the $G$-fixed category of $\mathcal{R}_{R}$ is the bimonoidal category associated to the $G$-fixed subring of $R$.

Example 6.7 For the category $\mathcal{V}_{\mathbb{C}}$ the $\mathbb{Z} / 2 \mathbb{Z}$-fixed category is the bipermutative category of real vector spaces, $\mathcal{V}_{\mathbb{R}}$, whose objects are again the natural numbers, but whose morphisms are given by

$$
\mathcal{V}_{\mathbb{R}}(n, m)= \begin{cases}\varnothing & n \neq m, \\ O(n) & n=m .\end{cases}
$$

Note that the homotopy fixed point spectrum $H \mathcal{V}_{\mathbb{C}}^{h \mathbb{Z} / 2 \mathbb{Z}}$ is $k u^{h \mathbb{Z} / 2 \mathbb{Z}}$ and this is not equivalent to the associated spectrum $k o=H \mathcal{V}_{\mathbb{R}}$. In the case of Eilenberg-Mac Lane spectra, however, we obtain that

$$
H R^{h G}=H \mathcal{R}_{R}^{h G} \simeq H\left(R^{G}\right)=H \mathcal{R}_{R^{G}} .
$$

Moreover, if $A \rightarrow B$ is a $G-$ Galois extension of commutative rings, then $H A=$ $H \mathcal{R}_{A} \rightarrow H \mathcal{R}_{B}=H B$ is a $G-$ Galois extension of commutative $S$-algebras in the sense of Rognes [17, Proposition 4.2.1].

Proposition 6.8 Let $\mathcal{R}$ be a (symmetric) bimonoidal category with $G$-action. Then the weak equivalence [3]

$$
\mathcal{K}(\mathcal{R}) \simeq K(H \mathcal{R})
$$

is $G$-equivariant.

Proof All constructions used in [3] are natural with respect to lax (symmetric) bimonoidal functors.

Remark 6.9 As $G$-actions on bimonoidal categories with anti-involution are given in terms of morphisms of such categories, they can be combined with the external involution on the bar construction for $\mathcal{K}(\mathcal{R})$. 


\section{Examples}

\subsection{Endomorphisms of a permutative category}

Let $\left(\mathcal{C}, \oplus, 0_{\mathcal{C}}, c_{\oplus}\right)$ be any permutative category. Consider the category of all lax symmetric monoidal functors from $\mathcal{C}$ to itself. Elmendorf and Mandell [11, page 176] describe how to impose a bimonoidal structure on this category. We denote this category by $\operatorname{End}(\mathcal{C})$. The addition is given "pointwise", ie, for two lax symmetric monoidal functors $F, G: \mathcal{C} \rightarrow \mathcal{C}$ one defines

$$
(F \oplus G)(C)=F(C) \oplus G(C) .
$$

The multiplicative structure is given by composition.

If we consider the full subcategory of $\operatorname{End}(\mathcal{C})$ of invertible lax symmetric monoidal functors and we take the bimonoidal subcategory of $\operatorname{End}(\mathcal{C})$ generated by these under direct sum and composition which we call $\operatorname{Inv}(\mathcal{C})$. One might think of $\operatorname{Inv}(\mathcal{C})$ as the group-rig of the category $\mathcal{C}$. We can define an involution on $\operatorname{Inv}(\mathcal{C})$ by sending an invertible functor $F$ to its inverse

$$
\zeta(F)=F^{-1}
$$

and extending this involution to finite words (under $\oplus$ and $\circ$ ) in such functors. For instance, we have

$$
\zeta\left(G_{1} \oplus G_{2}\right)=G_{1}^{-1} \oplus G_{2}^{-1}
$$

As we have

$$
(G \circ F)^{-1}=F^{-1} \circ G^{-1}
$$

we can choose $\mu$ to be the identity.

Group actions on (symmetric) bimonoidal categories provide nontrivial examples. If a discrete group $G$ acts on a (symmetric) bimonoidal category $\mathcal{R}$, then the elements of the group are objects of the category $\operatorname{Inv}(\mathcal{R})$. For instance the category of complex vector spaces $\mathcal{V}_{\mathbb{C}}$ with its $\mathbb{Z} / 2 \mathbb{Z}$-action gives rise to a nontrivial category $\operatorname{Inv}\left(\mathcal{V}_{\mathbb{C}}\right)$.

If $R$ is a ring with $G$-action, then the category $F(R)$ with objects $\mathbf{n} \in \mathbb{N}_{0}$ and morphisms the $R$-automorphisms of $R^{n}$ is a bimonoidal category with $G$-action. The action is trivial on objects and it sends an automorphism $\varphi$ to $g \varphi$ for $g \in G$ where $g \varphi$ is the morphism that sends $v \in R^{n}$ to $g \varphi(v)$. 


\subsection{Hopf bimodules}

Categories of Hopf bimodules provide a class of examples of (nonstrict) braided bimonoidal categories. Consider a Hopf algebra $H$ in a symmetric monoidal category. An object $M$ is an $H$ Hopf bimodule if it is a bimodule over $H$ and simultaneously an $H$ right- and left-comodule such that the comodule structure maps are morphisms of $H$-bimodules. Here, the diagonal on $H$ gives the $H$-bimodule structure on $H \otimes M$ and $M \otimes H$. Schauenburg showed [18, Theorem 6.3] that the category of $H$-Hopf bimodules, ${ }_{H}^{H} \mathcal{M}_{H}^{H}$, is a braided monoidal category with the tensor product over $H$, if the antipode of the Hopf algebra $H$ is invertible, and that the category ${ }_{H}^{H} \mathcal{M}_{H}^{H}$ is equivalent to the category of right Yetter-Drinfel'd $H$-modules [18, Theorem 5.7 (3)] if the underlying category has equalizers.

Let us consider the symmetric monoidal category of $k$-modules for some commutative ring $k$ with unit, with direct sum as the additive structure. Unadorned tensor products are tensor products over $k$. The category of $H$-bimodules, ${ }_{H} \mathcal{M}_{H}$, over a Hopf algebra $H$ is then a (non strict) bimonoidal category with the direct sum of $k$-modules as additive and the tensor product over $H$ as multiplicative structure. The direct sum of two $k$-modules $A, B \in{ }_{H} \mathcal{M}_{H}$ is an $H$-bimodule if we declare the structure maps to be

and

$$
H \otimes(A \oplus B) \stackrel{d_{\ell}^{-1}}{\longrightarrow} H \otimes A \oplus H \otimes B \longrightarrow A \oplus B
$$$$
(A \oplus B) \otimes H \stackrel{d_{r}^{-1}}{\longrightarrow} A \otimes H \oplus B \otimes H \longrightarrow A \oplus B .
$$

Here, $d_{\ell}$ and $d_{r}$ denote the distributivity isomorphisms in the underlying category of $k$-modules, ie,

$$
d_{\ell}: A \otimes B \oplus A \otimes B^{\prime} \rightarrow A \otimes\left(B \oplus B^{\prime}\right), \quad d_{r}: A \otimes B \oplus A^{\prime} \otimes B \rightarrow\left(A \oplus A^{\prime}\right) \otimes B .
$$

Similarly, the left and right comodule structures on $A$ and $B, \psi_{A}, \psi^{A}$ resp. $\psi_{B}, \psi^{B}$, give rise to a left and a right comodule structure on the sum via

$$
A \oplus B \stackrel{\psi_{A} \oplus \psi_{B}}{\longrightarrow} H \otimes A \oplus H \otimes B \stackrel{d_{\ell}}{\longrightarrow} H \otimes(A \oplus B)
$$

and

$$
A \oplus B \stackrel{\psi^{A} \oplus \psi^{B}}{\longrightarrow} A \otimes H \oplus B \otimes H \stackrel{d_{r}}{\longrightarrow}(A \oplus B) \otimes H .
$$

It is tedious but straightforward to check that the coherence isomorphisms of the bimonoidal category of $H$-bimodules are actually morphisms of comodules. The explicit form of the braiding from [18, Theorem 6.3] allows it to check that condition (7) of 
Definition 5.1 is indeed satisfied and that Laplaza's distributivity axioms [14, Section 1] are satisfied with the braiding $\beta$ replacing the multiplicative twist.

\subsection{Involutions on $A(*)$ and $A(B B G)$}

The associated spectrum of the bipermutative category $\mathcal{E}$ is the sphere spectrum and thus the equivalence from [3] identifies $\mathcal{K}(\mathcal{E})$ with the algebraic $K$-theory of the sphere spectrum, $K(S)$, which in turn is equivalent to Waldhausen's $A$-theory of a point, $A(*)$. Steiner constructed an involution on $A(X)$ for all spaces $X$ in [19, Theorem 3.10] where he used the model for $A(X)$ that consists of the algebraic $K-$ theory of the spherical group ring of $\Omega X, K(S[\Omega X])$. He defined his involution as the composition of loop inversion, matrix transposition and reversal of multiplication which in our context is taken care of by the reflection map on the bar construction. We do not provide a formal proof that Steiner's and our involution are equivalent in some suitable sense, but we just note that our definition resembles his. Another description of involutions on Waldhausen's $K$-theory of spaces is due to Vogell [20]. For a construction of spectrum level involutions on $S[\Omega M]$ for manifolds $M$ see Kro [13].

John Rognes drew my attention to the example of finite free $G$-sets and $G$-equivariant bijections. For a group $G$ we consider the following small version of this category. We define the category $\mathcal{E} G$ whose objects are again the finite sets $\mathbf{n}=\{1, \ldots, n\}$ for $n \in \mathbb{N}_{0}$ with $\mathbf{0}=\varnothing$ and whose morphisms are given by

$$
\mathcal{E} G(\mathbf{n}, \mathbf{m})= \begin{cases}\varnothing & n \neq m, \\ G \prec \Sigma_{n} & n=m .\end{cases}
$$

The classifying space $B(\mathcal{E} G)$ is

$$
\bigsqcup_{n \geqslant 0} B\left(G<\Sigma_{n}\right)=\bigsqcup_{n \geqslant 0} B G^{n} \times \Sigma_{n} E \Sigma_{n}
$$

For an abelian group $G$ we define a bipermutative structure on $\mathcal{E} G$ as follows. On objects, we take the bipermutative structure [2]), and on morphisms we define

$$
\left(g_{1}, \ldots, g_{n}, \sigma\right) \oplus\left(g_{1}^{\prime}, \ldots, g_{m}^{\prime}, \sigma^{\prime}\right)=\left(g_{1}, \ldots, g_{n}, g_{1}^{\prime}, \ldots, g_{m}^{\prime}, \sigma \oplus \sigma^{\prime}\right)
$$

for $\left(g_{1}, \ldots, g_{n}, \sigma\right) \in G \nmid \Sigma_{n}$ and $\left(g_{1}^{\prime}, \ldots, g_{m}^{\prime}, \sigma^{\prime}\right) \in G \succ \Sigma_{m}$.

There are natural isomorphisms

$$
c_{\oplus}^{G}:\left(g_{1}, \ldots, g_{n}, \sigma\right) \oplus\left(g_{1}^{\prime}, \ldots, g_{m}^{\prime}, \sigma^{\prime}\right) \rightarrow\left(g_{1}^{\prime}, \ldots, g_{m}^{\prime}, \sigma^{\prime}\right) \oplus\left(g_{1}, \ldots, g_{n}, \sigma\right)
$$


for all $\left(g_{1}, \ldots, g_{n}, \sigma\right)$ and $\left(g_{1}^{\prime}, \ldots, g_{m}^{\prime}, \sigma^{\prime}\right)$ that use the additive twist $c_{\oplus}$ from the structure of $\mathcal{E}$ and that shuffle the $g_{i}$ and $g_{j}^{\prime}$. It is straightforward to check, that $\left(\mathcal{E} G, \oplus, \mathbf{0}, c_{\oplus}^{G}\right)$ is a permutative category.

As a multiplicative structure we define

$$
\left(g_{1}, \ldots, g_{n}, \sigma\right) \otimes\left(g_{1}^{\prime}, \ldots, g_{m}^{\prime}, \sigma^{\prime}\right)=\left(g_{1} g_{1}^{\prime}, \ldots, g_{1} g_{m}^{\prime}, \ldots, g_{n} g_{1}^{\prime}, \ldots, g_{n} g_{m}^{\prime}, \sigma \otimes \sigma^{\prime}\right) .
$$

Note that for this construction to be functorial, the group $G$ has to be abelian. We can compare $\left(g_{1}, \ldots, g_{n}, \sigma\right) \otimes\left(g_{1}^{\prime}, \ldots, g_{m}^{\prime}, \sigma^{\prime}\right)$ with $\left(g_{1}^{\prime}, \ldots, g_{m}^{\prime}, \sigma^{\prime}\right) \otimes\left(g_{1}, \ldots, g_{n}, \sigma\right)$ by using natural isomorphisms $c_{\otimes}^{G}$ which are built out of the multiplicative twist $c_{\otimes}$ from $\mathcal{E}$ and which reorder arrays like $\left(g_{1} g_{1}^{\prime}, \ldots, g_{1} g_{m}^{\prime}, \ldots, g_{n} g_{1}^{\prime}, \ldots, g_{n} g_{m}^{\prime}\right)$ to $\left(g_{1}^{\prime} g_{1}, \ldots, g_{1}^{\prime} g_{n}, \ldots, g_{m}^{\prime} g_{1}, \ldots, g_{m}^{\prime} g_{n}\right)$.

With this multiplicative structure $\left(\mathcal{E} G, \otimes, \mathbf{1}, c_{\otimes}^{G}\right)$ is a permutative category and the multiplicative and additive structure combine to turn $\mathcal{E} G$ into a bipermutative category.

We can define an anti-involution on $\mathcal{E} G$ by declaring $\zeta$ to be the identity on objects and on morphisms we define $\zeta\left(g_{1}, \ldots, g_{n}, \sigma\right)=\left(g_{1}^{-1}, \ldots, g_{n}^{-1}, \sigma\right)$ for all $g_{i} \in G$ and permutations $\sigma$. Then $\zeta$ is strictly additive and we can use the multiplicative twist $c_{\otimes}^{G}$ in $\mathcal{E}$ as $\mu$ in order to obtain natural isomorphisms $\mu$ from $\zeta\left(\left(g_{1}^{\prime}, \ldots, g_{m}^{\prime}, \sigma^{\prime}\right) \otimes\right.$ $\left.\left(g_{1}, \ldots, g_{n}, \sigma\right)\right)$ to $\zeta\left(g_{1}, \ldots, g_{n}, \sigma\right) \otimes \zeta\left(g_{1}^{\prime}, \ldots, g_{m}^{\prime}, \sigma^{\prime}\right)$.

Barratt [6] defined his functor $\Gamma^{+}$for based simplicial sets $X$ and identified its geometric realization with $\Omega^{\infty} \Sigma^{\infty}|X|$. For $|X|=B G_{+}$we obtain that $\Omega^{\infty} \Sigma^{\infty} B G_{+}$ is the infinite loop space associated to the spectrum $H(\mathcal{E} G)$ and therefore this ring spectrum is the spherical group ring $S[B G]=\Sigma_{+}^{\infty}(B G)$. Its algebraic $K$-theory is Waldhausen's $K$-theory $A(B B G)=K(S[\Omega B B G]) \simeq K(S[B G])$.

For $G$ abelian, the inverse map on $G$ induces the inverse map on $B G$ and via the map of $H$-spaces $B G \stackrel{\sim}{\rightarrow} \Omega B B G$ this is related to loop inversion. In this sense the induced involution on $\mathcal{K}(\mathcal{E} G) \simeq A(B B G)$ is similar to Steiner's involution on $A(B B G)$.

\section{Nontriviality}

Farrell and Hsiang [12, Lemma 2.4] calculated the effect of the involution of $K_{i}(\mathbb{Z}) \otimes \mathbb{Q}$ : elements in positive degrees are sent to their additive inverse. We use this fact to prove the following.

Proposition 8.1 The involutions on $\mathcal{K}(\mathcal{V}) \simeq K(k u), \mathcal{K}\left(\mathcal{V}_{\mathbb{R}}\right) \simeq K(k o), \mathcal{K}(\mathcal{E} G) \simeq$ $A(B B G)$ ( $G$ abelian) are nontrivial. 
Proof We can model the map $\pi: k u \rightarrow H \mathbb{Z}$ of commutative ring spectra on the level of bipermutative categories $\pi: \mathcal{V} \rightarrow \mathcal{R}_{\mathbb{Z}}$ by sending an object $\mathbf{n}$ to the natural number $n$ and projecting the set $U(n)$ of endomorphisms of $\mathbf{n}$ to the set $\{\mathrm{id}\}$. This is a morphism of bipermutative categories with anti-involution. On the level of $K$-theory we obtain an induced map

$$
K(k u) \simeq \mathcal{K}(\mathcal{V}) \stackrel{\mathcal{K}(\pi)}{\longrightarrow} \mathcal{K}\left(\mathcal{R}_{\mathbb{Z}}\right) \simeq K^{f}(\mathbb{Z}) .
$$

Ausoni and Rognes show [1, Theorem 2.5 (a)] that rationally the map $K(\pi): K(k u) \rightarrow$ $K(\mathbb{Z})$ is split. As the involution is nontrivial on $K_{*>0}(\mathbb{Z}) \otimes \mathbb{Q}$, it is nontrivial on $K(k u)$. Similarly, they show that rationally $K(\mathbb{Z})$ splits off $K(k o)$.

Consider the following diagram of bipermutative categories with anti-involution:

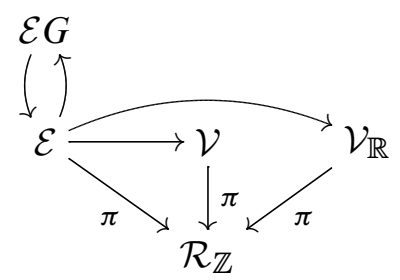

The maps from $\mathcal{E}$ model the unit map from the sphere spectrum $S \simeq H \mathcal{E}$ to $S[B G]$, $k o$ and $k u$ and are given by the identity on objects and the inclusion of $\Sigma_{n}$ into the respective endomorphisms of $\mathbf{n}$.

Rationally, $A(*) \simeq \mathcal{K}(\mathcal{E})$ agrees with $K(\mathbb{Z})$ and it splits off $A(B B G)$, so the involution is not trivial on $A(B B G)$.

Ausoni and Rognes also proved in [1] that rationally $A(K(\mathbb{Z}, 3))$ is equivalent to $K(k u)$. A map of ring spectra $A(K(\mathbb{Z}, 3)) \rightarrow K(k u)$ is given by using the string of maps

$$
B U(1) \rightarrow B U_{\otimes} \rightarrow \mathrm{GL}_{1}(k u) \rightarrow \Omega^{\infty}(k u)
$$

and taking the adjoint which is a map from the suspension ring spectrum $\Sigma_{+}^{\infty} B U(1) \simeq$ $S[B U(1)]$ to $k u$. This yields an induced map on algebraic $K$-theory $K(S[B U(1)]) \rightarrow$ $K(k u)$. We can model this via a functor of categories

$$
F: \mathcal{E} \mathbb{S}^{1} \rightarrow \mathcal{V}
$$

Here, $F$ sends $\mathbf{n}$ to $\mathbf{n}$ and maps a morphism $\left(z_{1}, \ldots, z_{n}, \sigma\right) \in \mathbb{S}^{1} \prec \Sigma_{n}$ to the matrix $\operatorname{diag}\left(z_{1}, \ldots, z_{n}\right) \cdot E_{\sigma} \in U(n)$ where diag denotes the corresponding diagonal matrix and $E_{\sigma}$ is the permutation matrix associated to $\sigma$. The fact that

$$
E_{\sigma} \cdot \operatorname{diag}\left(w_{1}, \ldots, w_{n}\right)=\operatorname{diag}\left(w_{\sigma^{-1}(1)}, \ldots, w_{\sigma^{-1} n}\right) \cdot E_{\sigma}
$$

for $w_{i} \in \mathbb{S}^{1}$ ensures the naturality of $F$. 
As the diagram

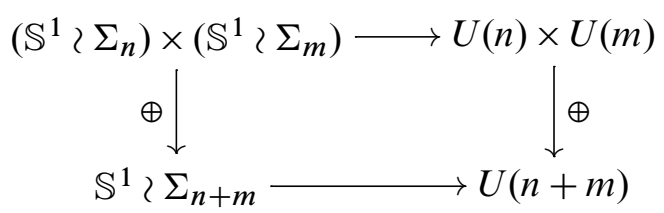

commutes, we see that $F$ respects addition. If $\left(e_{1}, \ldots, e_{n}\right)$ and $\left(f_{1}, \ldots, f_{m}\right)$ are ordered bases for $\mathbb{C}^{n}$ respectively $\mathbb{C}^{m}$, then we choose

$$
\left(e_{1} \otimes f_{1}, \ldots, e_{1} \otimes f_{m}, \ldots, e_{n} \otimes f_{1}, \ldots, e_{n} \otimes f_{m}\right)
$$

as an ordered basis for $\mathbb{C}^{n m}$. With this convention, $F$ respects $\otimes$ as well.

However, $F$ is not a functor of bimonoidal categories with anti-involution if we choose the anti-involution (id, $c_{\otimes}$ ) on $\mathcal{V}$ coming from its bipermutative structure.

Consider the $\mathbb{Z} / 2 \mathbb{Z}=\langle\xi\rangle$-action on $\mathcal{V}$ from Example 6.2.

Lemma 8.2 The composition $\bar{\zeta}$ of the anti-involution (id, $c_{\otimes}$ ) on $\mathcal{V}$ with the group action of $\mathbb{Z} / 2 \mathbb{Z}$ is an anti-involution on $\mathcal{V}$.

Proof If we set $\bar{\zeta}:=\xi \circ \zeta$, then

$$
\bar{\zeta} \circ \bar{\zeta}=\xi \circ \zeta \circ \xi \circ \zeta=\xi^{2} \circ \zeta^{2}=\mathrm{id}
$$

because $\xi$ and $\zeta$ commute. For two matrices $A \in U(n)$ and $B \in U(m)$ we have that $c_{\otimes}$ sends $\bar{\zeta}(A \otimes B)=\bar{A} \otimes \bar{B}$ to $\bar{B} \otimes \bar{A}$. The distributivity constraint from Definition 3.1 just express the fact that $d_{\ell}$ is given in terms of $d_{r}$ in $\mathcal{V}$. The remaining axioms are easy to check.

Corollary 8.3 The functor $F: \mathcal{E} \mathbb{S}^{1} \rightarrow \mathcal{V}$ is a morphism of bimonoidal categories with anti-involution

$$
F:\left(\mathcal{E S} \mathbb{S}^{1}, \zeta, c_{\otimes}^{G}\right) \longrightarrow\left(\mathcal{V}, \bar{\zeta}, c_{\otimes}\right)
$$

\section{References}

[1] C Ausoni, J Rognes, Algebraic K-theory of topological K-theory, Acta Math. 188 (2002) 1-39 MR1947457

[2] N A Baas, B I Dundas, B Richter, J Rognes, Ring completion of rig categories arXiv:0706. 0531

[3] N A Baas, B I Dundas, B Richter, J Rognes, Stable bundles over rig categories arXiv:0909.1742 
[4] N A Baas, B I Dundas, J Rognes, Two-vector bundles and forms of elliptic cohomology, from: "Topology, geometry and quantum field theory", (U Tillmann, editor), London Math. Soc. Lecture Note Ser. 308, Cambridge Univ. Press (2004) 18-45 MR2079370

[5] D Barnes, Rational equivariant spectra, $\mathrm{PhD}$ thesis, University of Sheffield (2008) arXiv:0802.0954

[6] M G Barratt, A free group functor for stable homotopy, from: "Algebraic topology (Proc. Sympos. Pure Math., Vol. XXII, Univ. Wisconsin, Madison, Wis., 1970)”, (A Liulevicius, editor), Amer. Math. Soc. (1971) 31-35 MR0324693

[7] D Burghelea, $\mathbf{Z}$ Fiedorowicz, Hermitian algebraic $K$-theory of simplicial rings and topological spaces, J. Math. Pures Appl. (9) 64 (1985) 175-235 MR820117

[8] S U Chase, D K Harrison, A Rosenberg, Galois theory and Galois cohomology of commutative rings, Mem. Amer. Math. Soc. No. 52 (1965) 15-33 MR0195922

[9] G Dunn, $K$-theory of braided tensor ring categories with higher commutativity, $K-$ Theory 9 (1995) 591-605 MR1361586

[10] G Dunn, $E_{n}-$ ring categories, J. Pure Appl. Algebra 119 (1997) 27-45 MR1456092

[11] A D Elmendorf, MA Mandell, Rings, modules, and algebras in infinite loop space theory, Adv. Math. 205 (2006) 163-228 MR2254311

[12] F T Farrell, W C Hsiang, On the rational homotopy groups of the diffeomorphism groups of discs, spheres and aspherical manifolds, from: "Algebraic and geometric topology (Proc. Sympos. Pure Math., Stanford Univ., 1976), Part 1”, (R J Milgram, editor), Proc. Sympos. Pure Math. XXXII, Amer. Math. Soc. (1978) 325-337 MR520509

[13] T A Kro, Involutions on $S[\Omega M]$, PhD thesis, University of Oslo (2005) arXiv: math.AT/0510221

[14] M L Laplaza, Coherence for distributivity, from: "Coherence in categories", (S Mac Lane, editor), Lecture Notes in Math. 281, Springer, Berlin (1972) 29-65 MR0335598

[15] S Mac Lane, Categories for the working mathematician, second edition, Graduate Texts in Math. 5, Springer, New York (1998) MR1712872

[16] J P May, $E_{\infty}$ ring spaces and $E_{\infty}$ ring spectra, Lecture Notes in Math. 577, Springer, Berlin (1977) MR0494077 With contributions by F Quinn, N Ray, and J Tornehave

[17] J Rognes, Galois extensions of structured ring spectra. Stably dualizable groups, Mem. Amer. Math. Soc. 192 (2008) viii+137 MR2387923

[18] P Schauenburg, Hopf modules and Yetter-Drinfel'd modules, J. Algebra 169 (1994) 874-890 MR1302122

[19] R J Steiner, Infinite loop structures on the algebraic $K$-theory of spaces, Math. Proc. Cambridge Philos. Soc. 90 (1981) 85-111 MR611287 
[20] W Vogell, The canonical involution on the algebraic $K$-theory of spaces, from: "Algebraic topology (Aarhus, 1982)", (I Madsen, B Oliver, editors), Lecture Notes in Math. 1051, Springer, Berlin (1984) 156-172 MR764578

Department Mathematik der Universität Hamburg Bundesstraße 55, 20146 Hamburg, Germany

richter@math . uni-hamburg.de

http://www . math.uni-hamburg.de/home/richter/

Received: 29 September 2008 Revised: 22 June 2009 